\title{
A DIRECT PROOF OF THE HOFMANN-MISLOVE THEOREM
}

\author{
KLAUS KEIMEL AND JAN PASEKA
}

(Communicated by Franklin D. Tall)

\begin{abstract}
The Hofmann-Mislove Theorem has attracted increasing interest. Through this note we intend to make it easily accessible by a simple direct proof.
\end{abstract}

Theorem. For every sober space $X$ there is an (order reversing) bijection between the set of Scott-open filters of the lattice $\mathcal{O}(X)$ of open subsets of $X$ and the set of quasicompact saturated subsers of $X$.

This theorem as well as the corollaries below were shown by Hofmann and Mislove in [2], with the help of the Spectral theory of continuous lattices (cf. [1, Chapter V]). More direct, but still in the language of locales, is the proof of Theorem 8.2.5 in [3]. Because of its importance, it seems worthwile to present a simple proof for the theorem which only uses standard general topology. However, we hasten to add that the underlying idea is essentially the same in all three presentations. But first, let us clarify the terminology.

First, a topological space $X$ is called sober if every irreducible closed subset of $X$ is the closure of some point $x$. Here a closed subset $C$ of $X$ is called irreducible if $C$ is nonempty and cannot be represented as a union of two of its proper closed subsets. Equivalently, the complement $U$ of $C$ is prime in the lattice $\mathscr{O}(X)$ of open subsets of $X$; i.e., $U$ is a proper open subset of $X$ that cannot be represented as an intersection of two strictly bigger open sets.

Second, let $\mathscr{F}$ be a filter in the lattice $\mathscr{O}(X)$, i.e., a collection of open subsets of $X$ such that

(i) $X \in \mathscr{F}$;

(ii) $U \in \mathscr{F}, U \subseteq V \in \mathscr{O}(X)$ imply $V \in \mathscr{O}(X)$; and

(iii) $U, V \in \mathscr{F}$ imply $U \cap V \in \mathscr{F}$.

Such a filter is called Scott-open (cf. [1, Chapter II]) if the following holds:

(O) Whenever $\left(U_{i}\right)_{i \in I}$ is a family of open sets such that $\bigcup_{i \in I} U_{i} \in \mathscr{F}$, then there are finitely many $i_{1}, \ldots, i_{n} \in I$ such that $\bigcup_{k=1}^{n} U_{i_{k}} \in \mathscr{F}$, or, equivalently,

Received by the editors October 23, 1991.

1991 Mathematics Subject Classification. Primary 54D30, 54D10.

Key words and phrases. Hofmann-Mislove Theorem, Scott-open filters.

The second author worked on this while he was visiting the Technische Hochschule Darmstadt under the auspices of the DAAD Foundation. 
$\left(\mathrm{O}^{\prime}\right)$ Whenever $\mathscr{D}$ is a directed family of open sets such that $\bigcup \mathscr{D} \in \mathscr{F}$, then $\mathscr{D} \cap \mathscr{F} \neq \varnothing$.

In the following, let $X$ always be a sober topological space. The proof of the Hofmann-Mislove Theorem relies on the following key lemma.

Lemma. Let $\mathscr{F}$ be a Scott-open filter in $\mathscr{O}(X)$. Then every open set $U$ containing $K=\bigcap \mathscr{F}$ is already a member of $\mathscr{F}$.

Proof. Assume that there is an open set $U$, which contains $K$ but which does not belong to $\mathscr{F}$. As $\mathscr{F}$ is Scott open, by Zorn's Lemma, there exists an open set $W$ which contains $U$ and which is maximal among all open sets not belonging to $\mathscr{F}$. Since $\mathscr{F}$ is a filter, $W$ is prime in $\mathscr{O}(X)$, which is equivalent to saying that the complement of $W$ is an irreducible closed set. We assumed $X$ to be sober. So the complement of $W$ is the closure of a point $x \in X$. Every open set $V$ which does not contain $x$ must be a subset of $W$. It therefore follows that $x$ is an element of every set $V$ belonging to $\mathscr{F}$. Of course, this implies $x \in \cap \mathscr{F}=K$, a contradiction to $W$ being a neighborhood of $K$.

Corollary 1. If $\mathscr{F}$ is a Scott-open filter in $\mathscr{O}(X)$, then $K=\bigcap \mathscr{F}$ is quasicompact; if, in addition, all the members of $\mathscr{F}$ are nonempty, then $K=\bigcap \mathscr{F}$ is nonempty, too.

Proof. Indeed, let $\left(U_{i}\right)_{i \in I}$ be an open covering of $K$; then $\bigcup_{i \in I} U_{i}$ is an open set containing $K$. Thus, $\bigcup_{i \in I} U_{i} \in \mathscr{F}$ by the Lemma. As $\mathscr{F}$ is Scott-open, there are $i_{1}, \ldots, i_{n}$ such that $U_{i_{1}} \cup \cdots \cup U_{i_{n}} \in \mathscr{F}$, i.e., $K \subseteq U_{i_{1}} \cup \cdots \cup U_{i_{n}}$, and we have shown that $K$ is quasicompact. If $K=\varnothing$, then $\varnothing \in \mathscr{F}$ again by the Lemma.

To every Scott-open filter $\mathscr{F}$ in $\mathscr{O}(X)$ we now have assigned a quasicompact subset $K=\bigcap \mathscr{F}$ of $X$. On the other hand, for any subset $K$ of $X$, the collection $\mathscr{U}(K)$ of all open neighborhoods of $K$ is a filter in $\mathscr{O}(X)$ which is easily seen to be Scott-open if $K$ is quasicompact. By the Lemma, we have $\mathscr{U}(\cap \mathscr{F})=\mathscr{F}$. But $\bigcap \mathscr{U}(K)$ may be bigger than $K$. Therefore we restrict ourselves to subsets $K$ which are intersections of open sets; such subsets are called saturated. This finishes the proof of the Hofmann-Mislove Theorem.

Finally, let us consider a family $\left(K_{i}\right)_{i \in I}$ of quasicompact saturated subsets of $X$ which is filtered (i.e., nonempty and for any $i, j \in I$ there exists an $l$ such that $\left.K_{l} \subseteq K_{i} \cap K_{j}\right)$. The union $\mathscr{F}$ of the Scott-open neighborhood filters $\mathscr{U}\left(K_{i}\right), i \in I$, clearly is a Scott-open filter again. Consequently, $\bigcap \mathscr{F}=\bigcap_{i \in I} K_{i}$ is also quasicompact. If all the $K_{i}$ are nonempty, their neighborhoods are nonempty, too, and we conclude that $\bigcap_{i \in I} K_{i}$ is nonempty, also by Corollary 1. Finally, if $U$ is an open set containing $\bigcap_{i \in I} K_{i}$, then $U \in \mathscr{F}$ by the Lemma, and consequently $U \supseteq K_{i}$ for some $i \in I$. So we have:

Corollary 2. Let $\left(K_{i}\right)_{i \in I}$ be a filtered family of nonempty quasicompact saturated subsets of $X$. Then $\bigcap_{i \in I} K_{i}$ is nonempty, quasicompact, and saturated, too; and an open set $U$ contains $\bigcap_{i \in I} K_{i}$ iff $U$ contains $K_{i}$ for some $i \in I$.

For Hausdorff spaces, Corollary 2 reduces to a simple but important basic fact about compact sets. It is the merit of Hofmann and Mislove [2] to have discovered its appropriate generalization to the non-Hausdorff case. It was the aim of this note to make their results easily accessible. 


\section{REFERENCES}

1. G. Gierz, K. H. Hofmann, K. Keimel, J. D. Lawson, M. Mislove, and D. S. Scott, $A$ compendium of continuous lattices, Springer-Verlag, Berlin, 1980.

2. K. H. Hofmann and M. Mislove, Local compactness and continuous lattices, Continuous Lattices (Proceedings, Bremen, 1979) (B. Banaschewski and R.-E. Hoffman, eds.), SpringerVerlag, Berlin, 1981, pp. 209-248.

3. S. J. Vickers, Topology via logic, Cambridge Univ. Press, Cambridge, 1989.

Fachbereich Mathematik, Technische Hochschule Darmstadt, Schlossgartenstrasse NR. 7, 64289 DARMSTADT, Germany

E-mail address: keimel@mathematik.th-darmstadt.de

Department of Mathematics, Masaryk University Brno, JanáčKovo nám. 2A, 66295 BRno, Czechoslovakia

E-mail address: paseka@adelard.dcs.muni.cs 